Bio-grafia: Escritos sobre la Biologia y su Enseñanza Vol 3 No4 ISSN 2027-1034. Primer semestre de 2010, Bogotá, Colombia, pp 55-71

\title{
Planteamientos en textos escolares de Ciencias Naturales relacionados con evolución por selección Natural, que pueden generar obstáculos Epistemológicos
}

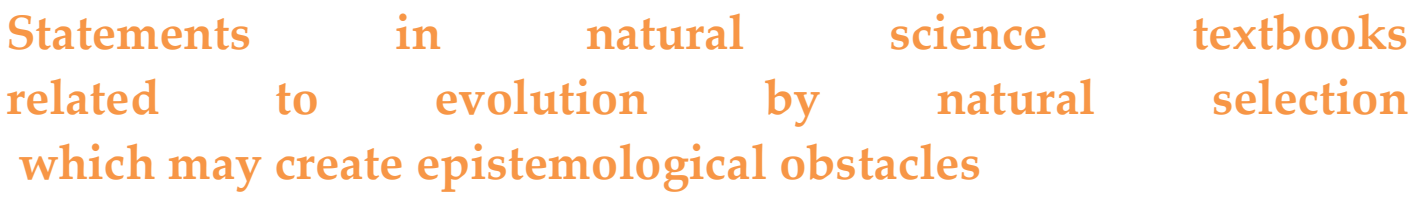

Por: Catalina Vallejo Ovalle ${ }^{1}$

\section{Resumen}

\begin{tabular}{|l|}
\hline Recibido: 01-04-2010 \\
\hline Aceptado: 22- 06-2010
\end{tabular}

El presente trabajo buscó indagar si existen, en algunos libros de texto usados en el nivel educativo de básica secundaria, planteamientos relacionados con el tema de evolución por selección natural, que podrían propiciar el surgimiento de obstáculos de tipo epistemológico en los estudiantes, y a la vez analizar su influencia en los procesos de enseñanza - aprendizaje de este tema en particular.

Esta investigación se enmarcó en una metodología cualitativa, empleando el análisis de contenido de tipo descriptivo, como técnica principal para detectar los planteamientos en los libros de texto objeto de estudio.

Este trabajo evidenció que existen planteamientos inadecuados en los libros de texto, que pueden propiciar la generación de obstáculos epistemológicos como: de conocimiento general, la explicación por la utilidad, el animista y el verbal. Siendo los más frecuentes el de conocimiento general, experiencia básica, el animista y el verbal. Además se encontró, que en los libros utilizados se tiende a simplificar los procesos que se dan en la evolución por selección natural, sin tenerse en cuenta las variables que hacen parte de ésta, ni las interrelaciones con el medio en que se desarrollan los organismos.

Palabras claves: Obstáculos epistemológicos, planteamientos, evolución, selección natural, textos escolares.

\section{Abstract}

This paper inquires into the existence of statements related to the topic of evolution by natural selection in some science texts, which are currently used in Basic Secondary, that could favour the appearance of epistemological obstacles in

Especialista en Enseñanza de la Biología. Asistente académica de un grupo de investigación del Doctorado Interinstitucioal en Educación. Universidad Pedagógica Nacional. Universidad Pedagógica Nacional 
students, and at the same time, analyze their influence in the teaching and learning processes; specially in this topic.

This investigation is framed in a qualitative methodology, using the content descriptive analysis as the main technique to detect statements in the studied textbooks.

This work evidenced the existence of inadequate statements in the analyzed textbooks that may propitiate the raise of epistemological obstacles like those of general knowledge, explanation for utility, animism and verbal. Being the most frequent the one of general knowledge, basic experience, animist and verbal. It was also found that the used textbooks tend to simplify the processes that take place in evolution by natural selection without taking into account the variables that make part of this process, neither the interrelations that occur with the environment where organisms develop.

Key words: Epistemological obstacles, statements, evolution, natural selection, textbooks.

\section{INTRODUCCIÓN}

Este estudio hace un aporte a la enseñanza, en el sentido en que propicia la reflexión en torno al uso que hacen los maestros de la Información presentada en los libros de texto al momento de orientar el trabajo con los estudiantes, así mismo, esta investigación al centrarse en el tema evolución, resulta importante debido a que es uno de los conceptos estructurantes ${ }^{2}$ de la biología como lo señala Dobzhansky (1980:35) al plantear: "Nada tiene sentido en biología si no es a la luz de la evolución" y Mayr (1980), al afirmar que es importante a la hora de hablar de enseñanza de la biología, puesto que si los estudiantes tienen claridad en este concepto, podrán establecer más fácilmente relaciones con otros temas que parten de los postulados del concepto de evolución.

Según Cuellar et. al (2005) en el sistema educativo Colombiano se ha encontrado que el libro de texto, es el material más usado como modelo de currículo en las instituciones educativas. Los docentes utilizan en la preparación de sus clases de manera fundamental los libros de texto que usan los estudiantes y algunas veces aquellos que son diseñados para los maestros, textos que en ocasiones no contienen el sustento teórico y metodológico necesario para orientar los procesos de enseñanza aprendizaje de un tema, debido a que en el trabajo de síntesis que realizan las editoriales, en algunos casos se hace una simplificación de las temáticas, situación que no es del todo negativa porque se supondría que el maestro complementaría su desarrollo, pero cuando esto no es así se corre el riesgo de propiciar planteamientos que podrían llevar al surgimiento de obstáculos de tipo epistemológico, lo cual conduciría a un estancamiento en el aprendizaje.

Siendo consciente de que la información presente en los libros de texto no es la única causa que puede llevar a la formación de obstáculos epistemológicos, se puede decir que los

${ }^{2}$ Son conceptos que van a transformar el sistema cognitivo del alumno de tal manera que le van a permitir, de una forma coherente, adquirir nuevos conocimientos, por construcción de nuevos significados, o modificación de los anteriores, por reconstrucción de significados antiguos (García, 1998). 
conocimientos previos, el profesor, los medios de comunicación y el ambiente en el cual se desarrollan los estudiantes favorece o no el arraigo de algunas ideas que pueden dificultar la comprensión de algunos conceptos.

En este trabajo se buscó identificar qué planteamientos presentes en algunos libros de texto de ciencias naturales, potencialmente podrían favorecer el surgimiento de obstáculos de tipo epistemológico en estudiantes del grado noveno de la educación básica secundaria en Colombia, al momento de comprender el concepto de evolución por selección natural.

Para el desarrollo de esta investigación fue necesario abordar algunos referentes conceptuales que se describen a continuación:

Gastón Bachelard en la década de los años treinta, trabajó un problema que tuvó gran impacto en las teorías del conocimiento, observó que los estudiantes escolares en física tenían grandes dificultades para comprender el principio de Arquímedes; no se trataba de un problema de retención de datos, sino de comprender el principio mismo. Sus estudios demostraron que muchos estudiantes podían repetir mecánicamente sus lecciones, pero con el paso del tiempo o con una profundización de un tema, la calidad del aprendizaje disminuía, llegando Bachelard a la conclusión, de que los estudiantes o no comprendían o repetían simplemente las lecciones. Se trataba de un problema sencillo pero significativo, existía un choque de modelos mentales; esto se llamó obstáculo epistemológico (Hernández, 2003).

Según Hernández (2003) los trabajos sobre las concepciones de obstáculos, en Bachelard y Piaget, muestran que el error y el fracaso, no tienen origen simplificado como a veces se quiere hacer ver, según ellos el error no es solamente el efecto de la ignorancia, de la incertidumbre o el azar, sino el efecto de un conocimiento anterior que en determinado momento pudo ser adecuado, pero que en el momento actual no lo es; estos errores se constituyen en obstáculos y se encuentran tanto en el maestro como en el estudiante. Bachelard (1948) define el obstáculo epistemológico como, "lo que se sabe y que, como ya se sabe, genera una inercia que dificulta el proceso de construcción de un saber nuevo que es, precisamente lo que constituye el acto de conocer".

Tipos de obstáculos epistemológicos según Mora (2005):

- La experiencia básica o conocimientos previos: Hace referencia a aquellos conocimientos que el sujeto posee producto del contexto en que se desenvuelve.

- El obstáculo verbal: Se presenta cuando se quiere explicar un concepto por medio de una sola palabra o imagen.

- El peligro de la explicación por la utilidad: Se refiere a la tendencia de hallarle siempre utilidad al conocimiento.

- EI conocimiento general: Tendencia a explicar un concepto mediante el uso de generalizaciones.

- El obstáculo animista: Tendencia a explicar algunos hechos, fenómenos u objetos a partir de analogías con la naturaleza animada.

También se retoma el término planteamiento inadecuado, para hacer referencia a aquella información relacionada con el tema de la evolución por selección natural, que se encuentra en 
los libros de texto trabajados en las clases de ciencias y que potencialmente pueden llegar a generar Obstáculos Epistemológicos.

En relación a los libros de texto, Alzate (2000) usa el término, para hacer referencia a los libros escritos, diseñados y producidos específicamente para el uso en secuencias de enseñanza.

\section{MATERIALES Y MÉTODOS}

Este trabajo se orientó desde el enfoque de investigación cualitativa, la cual, según Arnal (1992) permite al investigador la descripción, comprensión e interpretación de los fenómenos propios de la realidad social, en este caso de los contextos educativos.

Como técnica principal se utilizó el análisis de contenido, que según Piñuel (2002) es el conjunto de procedimientos interpretativos de productos comunicativos (mensajes, textos o discursos) que proceden de procesos singulares de comunicación, teniendo por objeto elaborar y procesar datos relevantes sobre las situación en que se han producido aquellos textos, o sobre las condiciones que puedan darse para su empleo posterior. Esta técnica establece un vínculo entre tres niveles del lenguaje: el primero es el nivel superficial en el cual se hace la descripción de la información recopilada. En el segundo se realiza un análisis en el cual se intenta clasificar y ordenar la información y construir categorías y en el tercero se pretende interpretar para comprender y construir sentidos (Jiménez y Torres, 2004).

Este estudio se llevó a cabo en tres fases. En la primera fase se realizó la búsqueda y la recopilación de fuentes de información, que aportaron sustentos teóricos y metodológicos para el diseño de la investigación.

La segunda fase se dividió en tres etapas. En la primera se seleccionaron los libros de texto a utilizar. En la siguiente etapa se seleccionaron las categorías de análisis; para su formulación se realizó un ejercicio de revisión teórica, a través del cual se determinaron elementos constitutivos del tema de evolución por selección natural, esto permitió formular las categorías iníciales que fueron las siguientes: Tiempo geológico, Diversidad, Cambio, Adaptación, Antepasados, Descendencia-reproducción y Selección Natural. Cabe resaltar que en el proceso de análisis surgieron otras tres categorías que inicialmente no estaban definidas, estas son: Teleología y Observación. En la tercera fase, se seleccionaron frases contextualizadas como unidades de análisis, es decir se tuvieron en cuenta las frases para incluirlas dentro de las categorías, pero sin desconocer el contexto en el cual están inmersas para lograr una mejor interpretación. También se eligió el sistema de codificación que constó de tres partes: una letra y el número correspondiente al libro de texto, una letra mayúscula de la categoría y un número de la unidad de análisis. 
En la tercera fase del proyecto se realizaron los análisis y la síntesis interpretativa, los cuales brindaron elementos para hacer algunas recomendaciones en cuanto al uso de textos escolares al momento de trabajar el tema de evolución por selección natural. Y por último se generaron algunas conclusiones producto del trabajo realizado.

\section{RESULTADOS Y DISCUSIÓN}

\section{Categorías de análisis}

Las categorías de análisis usadas en esta investigación fueron de dos tipos unas iníciales ${ }^{3}$ y otras emergentes, es de resaltar que cada una de las categorías fue definida de acuerdo a la revisión teórica realizada durante la investigación. Las primeras categorías fueron definidas a partir de una revisión histórica y epistemológica del desarrollo del pensamiento evolutivo, en el cual se identificaron los conceptos fundamentales al momento de abordar la teoría de la evolución por selección natural y las segundas surgieron durante el desarrollo de la investigación, siendo estas transversales en los planteamientos de los libros de texto. Cabe anotar que algunos planteamientos identificados pueden ser ubicados en varias categorías, ante lo cual se procedió a clasificarlos según su vinculación más directa.

A continuación se enuncian las categorías y los autores que se retomaron como base para definirlas:

\section{Categorías iníciales}

\begin{tabular}{|l|l|}
\hline \multicolumn{1}{|c|}{ Categoría } & \multicolumn{1}{|c|}{ Autores } \\
\hline $\begin{array}{l}\text { Tiempo geológico, entendido como una } \\
\text { escala de tiempo que abarca los eventos } \\
\text { relacionados con la evolución de la vida } \\
\text { sobre la tierra. }\end{array}$ & $\begin{array}{l}\text { Eldredge, N (1985 a y b) en } \\
\text { Badii, M. et al (2008). } \\
\text { Rojas, O. (2008) }\end{array}$ \\
\hline $\begin{array}{l}\text { La diversidad, se asume como la variedad } \\
\text { de los organismos, producto de millones de } \\
\text { años de evolución. }\end{array}$ & Mayr, E. (2005) \\
Bidau, C. (2001) \\
\hline $\begin{array}{l}\text { El cambio, se comprende como aquellas } \\
\text { transformaciones que sufren las poblaciones } \\
\text { y los individuos a través del tiempo y el } \\
\text { espacio. }\end{array}$ & Bunge, M. (2005) \\
\hline
\end{tabular}

Asumidas como medios que permitieron relacionar aspectos teóricos, con lo encontrado en los libros de texto 


\begin{tabular}{|l|l|}
\hline $\begin{array}{l}\text { La adaptación es entendida como un proceso } \\
\text { gradual que desarrollan los organismos a nivel } \\
\text { de su morfología, fisiología y comportamiento }\end{array}$ & Ayala, F. (2006) \\
$\begin{array}{l}\text { y que les permite mantenerse con vida en un } \\
\text { determinado ambiente. }\end{array}$ & \\
\hline $\begin{array}{l}\text { Antepasados, aquella característica que hace } \\
\text { que las especies se vean ligadas entre sí, por } \\
\text { líneas de afinidad, que se remontan al } \\
\text { pasado evolutivo a través de una gran } \\
\text { cantidad de predecesores. }\end{array}$ & Reece, J. (2001) \\
\hline $\begin{array}{l}\text { La descendencialreproducción, se asumen } \\
\text { como mecanismos capaces de prolongar la } \\
\text { existencia de un organismo en el espacio y en } \\
\text { el tiempo. }\end{array}$ & Monje, J. et. al (2005) \\
\hline $\begin{array}{l}\text { La selección natural, es asumida como el } \\
\text { proceso y resultado de la supervivencia de } \\
\text { unos organismos con respecto a otros en un } \\
\text { determinado tiempo y ambiente especifico. }\end{array}$ & Ayala, F. (2006) \\
\hline
\end{tabular}

\section{Categorización de la información}

Para obtener la información se seleccionaron tres libros de ciencias naturales utilizados en grado noveno de la básica secundaria, debido a que por razones de organización curricular es en este grado escolar donde se trabaja el tema de evolución. Dichos libros fueron asumidos como muestra representativa de acuerdo a los siguientes criterios: los más usados en el sistema educativo y los más vendidos (previa consulta con algunas editoriales y puntos de venta).

Metodológicamente cada libro fue denominado con una letra y un número el cual, lo identificó en el proceso de análisis, los libros fueron denominados como L1, L2 y L3. A continuación se hace una breve descripción de cómo se aborda el tema de estudio en cada uno de los libros:

(L1): En este libro el tema de la evolución hace parte de la unidad tres, denominada "Historia de la teoría de la evolución", la cual, se subdivide en: una parte histórica titulada "Historia de la ciencia, el viaje de Darwin" en la que se hace un breve recorrido en torno al viaje realizado por Darwin en el Beagle. Posteriormente se presenta un subtitulo denominado "Darwinismo" en donde se tratan aspectos relacionados con el pensamiento de Darwin, éste a su vez se subdivide en otros numerales, titulados "Evolución por selección natural", "Selección natural y los pinzones de Darwin" y "Neodarwinismo" apartes utilizados para realizar el análisis de contenido. En esta unidad también se abordan temáticas como: Las evidencias de la evolución, Fisiología y bioquímica y Paleontología. En este libro se presentan actividades 
complementarias, en las cuales se retoman algunos de los aspectos trabajados en el tema de evolución por selección natural.

(L2): El tema de la evolución hace parte de la unidad dos denominada "Investiguemos los Seres de la Naturaleza". En dicho tema se abordan inicialmente las ideas de Lamarck, luego se realiza una contextualización del trabajo realizado por Darwin y se plantea el subtitulo "Darwin y la teoría de la evolución por selección natural", posteriormente se desarrolla un aparte titulado "Evidencias de la evolución". Planteando el siguiente estándar de contenido para esta temática: Identificar los principios de la evolución. Al finalizar el tema se retoma algunos aspectos del contenido trabajado, específicamente en la relación con la evolución por selección natural y plantean algunas preguntas abiertas relacionadas con la temática.

(L3): En este libro el tema de evolución se encuentra incluido en la unidad dos, titulada "Diversidad biológica", en la cual se trabajan las siguientes temáticas: Teoría de la evolución, La selección natural, Fenotipo y genotipo, analogía y homología, Jerarquías taxonómicas, especie, caracteres taxonómicos y reinos de la naturaleza. Para trabajar la temática de la teoría de la evolución se plantean los siguientes logros: Reconocer que los cambios de las especies dependen del código genético y del medio ambiente y describir la historia de la teoría evolutiva y la concepción actual del darwinismo. Al finalizar la unidad se plantean algunas preguntas de selección múltiple relacionadas con el tema.

\section{Interpretación de las categorías de análisis}

Teniendo en cuenta las categorías propuestas inicialmente, a continuación se identificaron en cada libro, los planteamientos que pueden llevar potencialmente al surgimiento de obstáculos epistemológico, al momento de acercarse a la construcción del concepto evolución por selección natural. Cabe aclarar que sólo se retomaron las unidades de análisis consideradas como planteamientos inadecuados, las demás fueron tenidas en cuenta como contexto.

A continuación se presentarán algunos de los planteamientos identificados para cada categoría:

\section{Categoría Tiempo Geológico (A)}

En relación con la categoría tiempo en el código L1A2 se plantea "...si algunas características de una población fueran seleccionadas por varias generaciones toda la población finalmente presentaría esas características...", podemos decir que sería apresurado afirmar que toda la población tendría determinadas características, no se puede olvidar que las mutaciones son en gran medida azarosas, no responden a principios, son independientes del proceso de selección, por lo tanto no todos los individuo de las poblaciones tendrían exactamente las mismas características. Se podría decir que habría una tendencia a poseer cierta característica, además es importante que esta idea no se plantee como una afirmación y como algo intencionado, ya que se puede prestar para una comprensión inadecuada.

En relación con el planteamiento identificado en L1, se tiende a hacer generalizaciones y afirmaciones, haciendo que los conceptos se tornen en verdades que no dan lugar a posicionamientos críticos por parte del estudiante, esto lejos de ser útil para el aprendizaje de las ciencias se convierten un obstáculos, porque inmovilizan el pensamiento; a partir de estas 
características lo podríamos clasificar como un obstáculo epistemológico de conocimiento general.

\section{Categoría Diversidad (B)}

En esta categoría en L2B1, se realiza la siguiente afirmación"...los individuos varían porque heredan diferentes caracteres..." aspecto que resulta acorde con la teoría de la evolución por selección natural, pero presentado de esta forma, podrían limitar la consideración de otros factores al momento de entender las diferencias entre los individuos, ya que se dejan de lado otras variables que también pueden influir en este proceso como es el ambiente en el que se desarrollan los individuos. Según Jacob (1986) cuanto más se diversifican los miembros de un grupo, por su conformación, sus propiedades y costumbres, en mejores condiciones se encuentran para ocupar hábitats variados y adaptarse a ellos.

En L2, encontramos planteamientos que podrían contribuir a la generación de obstáculos de tipo de conocimiento general, debido a que se hacen afirmaciones que pueden resultar sencillas, pero que en determinado momento pueden llegar a dificultar la compresión y aprendizaje del concepto. Según Mora (2005) al explicar mediante el uso de generalizaciones un concepto o una temática, en la mayoría de veces se cae en equivocaciones porque los conocimientos se vuelven vagos e indefinidos, ya que se dan definiciones tan amplias, dejando de lado aspectos esenciales y detalles que son los que permiten exponer con claridad las temáticas.

\section{Categoría Cambio (C)}

En el código L1C1 se afirma lo siguiente “...Si algunas características de una población fueran seleccionadas por varias generaciones, toda la población finalmente tendría esas características..."en relación con esta afirmación sería conveniente hacer la aclaración que no es toda la población, sino que la mayoría de individuos de esa población tendría dichas características debido a que pueden existir variaciones azarosas en los individuos que conforman las poblaciones.

En relación con esta categoría en L1 se encuentra que puede llevar al estudiante generar un obstáculo de tipo de conocimiento general, debido a que se hacen afirmaciones, que dejan de lado detalles o precisiones importantes, que pueden inducir a errores de conceptualización, con explicaciones sencillas aplicadas a todo una temática de forma resumida y concreta, lo cual va a dificultar su proceso de construcción de conocimiento.

En el código L2C2 se dice "... Darwin comprobó la lucha por la existencia en todas las poblaciones y concluyó que en esta lucha se conservan las variaciones favorables y se eliminan las desfavorables...", se hace alusión a la palabra lucha la cual puede ser entendida como sinónimo de disputa, lo cual en el proceso de la selección natural no siempre es cierto, debido a que muchas veces los organismos que aparentemente no luchan son los que permanecen, Si esta metáfora es interpretado textualmente puede convertirse en un problema, pero si su comprensión es orientada por el docente puede ser usada adecuadamente y facilitar la comprensión. 
Este planteamiento pueden llevar al estudiante a generar un obstáculo epistemológico de tipo verbal, debido a que se utiliza una palabra propia de las dinámicas humanas como recurso didáctico para explicar un proceso natural, convirtiéndose ésta en una barrera para el aprendizaje. Según Camilloni (1997) "las palabras invaden el campo de las ciencias, la ciencia se expresa en palabras y los conceptos de un campo remiten a los significados de los términos que se han construido en otras redes semánticas, pero no conservan su carácter extranjero, lo cual puede llevar a comprensiones erróneas".

\section{Categoría Adaptación (D)}

En L2D1 se plantea"...Todo organismo sostiene una lucha constante para sobrevivir..." el termino "lucha", tiene un carácter antropomórfico, que puede llevar a los estudiantes al imaginario de una pelea entre los organismos, ésto podría ser una dificultad porque cargaría de intencionalidad un hecho que sucede en los organismos. Sería entonces necesario hacer énfasis en la diferencia que existe entre que los organismos luchen y otra en que éstos sean conscientes de que luchan.

La supervivencia del más apto o del más fuerte, no es la mejor descripción de lo que ocurre en la naturaleza, a veces no existe un tipo más apto, pueden haber varios tipos diferentes que están igualmente adaptados por diferentes razones, quizás ellos están adaptados a diferentes facetas del ambiente, uno no va a remplazar al otro porque cada uno tiene su propio lugar en el ambiente. Además no es una función de supervivencia exclusivamente, la selección natural es una diferencia en el éxito reproductivo, el cual involucra tanto la habilidad de sobrevivir hasta la edad reproductiva como la capacidad de reproducirse.

En L2D2 se afirma"...Cada organismo debe luchar por la comida, el espacio, el agua, etc.; es decir, compite con nosotros..." siendo el mismo texto vuelve a nombrarse la palabra lucha anteriormente analizada reforzando este planteamiento y por otro lado se presenta como si todos los organismos tuvieran una relación directa con el hombre, siendo esta afirmación poco apropiada debido a que no todos los organismos compiten de forma directa con éste.

En relación con el código L2D3 se presenta la siguiente afirmación “...Esta competencia es más intensa entre los organismos de la misma especie pues tienen las mismas necesidades. Sobreviven quienes ganan la competencia..." Se hace un uso inapropiado de las palabras "ganan" y "competencia", es el hombre el que da esta caracterización, no se trata de ganar una competencia sino de la posibilidad de adaptarse y reproducirse exitosamente.

Tanto en L2D1, L2D2 y L2D3, se encontraron planteamientos que pueden llevar a la generación de obstáculos de tipo verbal y animista. Verbal debido a que mediante una palabra en este caso "lucha" se quiere explicar toda una idea, esto debido a que a veces se piensa que la función de la palabra, es de una evidencia tan clara que no se siente la necesidad de explicarla. Es así, como los estudiantes podrían llegar a pensar que la evolución por selección natural consiste en una lucha, simplificando todo un desarrollo teórico que se ha dado alrededor de este tema, restándole complejidad e importancia a un tema que al ser estructurante en la biología va a afectar la comprensión de otras temáticas derivadas o que parten de estos planteamientos. 
En cuanto al Obstáculo animista, se encuentra que estas afirmaciones están cargadas de una intencionalidad, como si todos los organismo se propusieran luchar para sobrevivir o luchar para competir específicamente con el hombre, lo cual sólo da argumentos para afirmar que se tiende a interpretar el mundo, luchas y pasiones propias del hombre, limitando así la comprensión de otras formas de vida. También podemos decir que el autor de este texto tiende a antropomorfizar las dinámicas propias de la naturaleza, siendo evidente que en él está presente el obstáculo del animismo el cual puede ser reproducido en los estudiantes que lean el texto.

\section{Categoría Antepasados (E)}

En el código L3E1 se plantea "...Para Darwin, los organismos semejantes están emparentados y descienden de un antepasado común..." si bien es cierto que los organismos semejantes y siendo de la misma especie, tendrían un antepasado común, sería importante aclarar que en algunos casos los organismos siendo semejantes morfológicamente, no necesariamente descienden de un antepasado común.

En relación con L3 podemos observar un planteamiento que puede contribuir a generar obstáculos de experiencia básica y conocimiento general. Experiencia básica, debido a que el estudiante tenderá a pensar que todo lo que se parezca a simple vista (morfológicamente), tiene un origen común. De acuerdo con Camillioni (1997:15) "los datos sensoriales netos oscurecen el conocimiento porque inducen a pensar que se conocen los objetos tal y como los vemos". Esto carga de subjetividad las observaciones ya que las cosas se ven tal como las observamos y no como realmente son, es decir, se elimina todo crítica, toda posibilidad de duda, lo cual va en una dirección opuesta a lo que debería ser el conocimiento científico, que según autores como Bachelard (1984) y Camilloni (1997) no puede construirse sin critica.

Esta afirmación también se convierte en un obstáculo de conocimiento general porque se realizan generalizaciones, debido a que la única razón encontrada simplifica la explicación, lo cual empobrece en consecuencia el pensamiento del estudiante, es decir, al afirmar que "los organismos semejantes están emparentados y descienden de un antepasado común" se dejan de lado toda una serie de ideas que le restan complejidad y claridad a este planteamiento, limitando la adecuada comprensión de la idea.

\section{Categoría Descendencia (reproducción) (F)}

En el código L2F1"...Todos los organismos producen más descendientes de los que en realidad pueden sobrevivir..." esta generalización puede ser imprecisa, ya que si revisamos los grupos de organismos no todos presentan estas características por lo tanto seria importante hacer la aclaración. Autores como Curtis (2008:339) hacen referencia a este aspecto como una de las premisas básicas presentadas por Darwin en su libro El Origen de las especies, pero lo plantea de la siguiente forma: "En la mayoría de las especies, el número de descendientes que sobreviven y se reproducen en cada generación es menor que el número inicial de descendientes" visto de esta forma da a entender que existen excepciones en relación con esta afirmación, dando espacio para que el estudiante se cuestionen e investigue acerca de cuales serían las especies que no presentan este tipo de comportamiento, abriendo así el debate en relación con este tema. 
En L2F1 encontramos afirmaciones que pueden llevar al estudiante a un obstáculo epistemológico de conocimiento general, debido a que se hacen generalizaciones y afirmaciones presentadas como verdades que no favorecen ni promueven el pensamiento crítico en los estudiantes. Como lo asegura Camilloni (1997:16) "ninguna afirmación en el discurso de las ciencias es definitivo, ni exento de dudas", además si la ciencia exige crítica y es un sistema en que el conocimiento cambia en el transcurso del tiempo, toda aserción debe ser revisada en el contexto de la teoría que la sustenta (Camilloni 1997).

En el código L2F4"...Los progenitores que sobreviven gracias a ciertos caracteres útiles, le transmiten éstos a su descendencia. La descendencia que aparezca con estos caracteres tendrán más posibilidad de sobrevivir..." en esta afirmación se hace alusión en términos utilitaristas e intencionales, a los caracteres presentes en los organismos, los cuales tienen un componente aleatorio y otro elemento direccional.

Este planteamiento puede llevar a un obstáculo de explicación por la utilidad, debido a que en la afirmación se evidencia una tendencia a reducir y sintetizar un concepto, solamente mediante la idea de utilidad o beneficio. Según Bachelard (1984: 87) "En todos los fenómenos se busca la utilidad, no sólo por la ventaja positiva que pueda procurar sino como principio de explicación" esto lleva al estudiante a dar explicaciones finalistas y en algunos ocasiones sin rigor científico.

\section{Categoría Selección natural (G)}

En relación con esta categoría en el código L3G1 “...Para Darwin, los organismos semejantes están emparentados y descienden de un antepasado común; afirma también que la evolución es producto de la selección natural, a través de la sobrevivencia en la lucha por la existencia..." en esta afirmación se plantean aspectos que fueron analizados anteriormente como es el caso de la semejanza entre los organismos emparentados y que descienden de un antepasado común. Otra parte de ésta afirmación es “...la evolución es producto de la selección natural, a través de la sobrevivencia en la lucha por la existencia..." en la cual se plantea como si la selección natural consistiera en una lucha intencional (consciente) por parte de los organismos, lo cual como ya se ha dicho, es contrario a la teoría de la evolución por selección natural, ya que ésta lo que hace es "escoger" las variaciones aleatorias a través de la interacción de los organismos individuales con su ambiente. Además es importante aclarar que aunque la selección natural es el principal proceso por el cual se da la evolución no se puede olvidar que también es producto de la deriva genética, la recombinación y el flujo genético.

En esta afirmación encontramos un planteamiento que pueden llevar a los estudiantes a crear obstáculos epistemológicos de tipo animista y verbal. Animista debido a que se carga de una intencionalidad al proceso de selección natural, lo cual como ya se ha expuesto es contrario a la teoría. En relación con el obstáculo verbal se hace uso simplificado de palabras en este caso "lucha" creyendo según Mora (2005) que "al asociar una palabra concreta a una palabra abstracta se hace avanzar el pensamiento, cuando en realidad lo que se ha presentado es un movimiento puro y simplemente lingüístico" que puede hacer pensar al estudiante que comprende lo que expone el libro de texto cuando en realidad lo que hace es asumir y repetir acríticamente, una "verdad" expresada por el autor en el libro. 


\section{CATEGORÍAS EMERGENTES}

En el transcurso del proceso investigativo se evidenciaron dos categorías, que no se habían tenido en cuenta inicialmente, estas son: teleología ${ }^{4}$, y observación ${ }^{5}$, categorías que pueden influir de manera significativa en la construcción que los estudiantes realicen sobre la teoría de la evolución por selección natural. Estas enmarcan el desarrollo de este trabajo, debido a que son postulados que buscan dar explicación a la evolución y que son trasversales en los planteamientos que se hacen en los tres libros de texto utilizados.

\section{Categoría Teleología}

Esta categoría resulta relevante debido a que a través del análisis se evidenció una marcada tendencia a hacer alusión a los fenómenos relacionados con la teoría de la evolución por selección natural, a partir de afirmaciones que daban a entender que este proceso se orientados hacia un progreso, intencionalidad o perfección de los organismos. Estas afirmaciones fueron relacionadas con el obstáculo epistemológico animista.

Para esta categoría tanto en L1, L2 y L3 encontramos planteamientos que pueden dar a entender que en los seres vivos existe un propósito o una finalidad.

En L1 se realizan las siguientes afirmaciones:

“...Si algunas características de una población fueran seleccionadas por varias generaciones, toda la población finalmente tendría esas características...".

"...Las características seleccionadas serían aquellas que le permitirían a los organismos una mejor supervivencia y mayores posibilidades de reproducirse, y los individuos con características adversas serian aquellos que morirían sin reproducirse...".

“...Para Darwin, los organismos semejantes están emparentados y descienden de un antepasado común; afirma también que la evolución es producto de la selección natural, a través de la sobrevivencia en la lucha por la existencia...".

En L2 se presentan las siguientes afirmaciones:

“...Todo organismo sostiene una lucha constante para sobrevivir...",

“...Cada organismo debe luchar por la comida, el espacio, el agua, etc.; es decir, compite con nosotros...",

"...y concluyó que en esta lucha se conservan las variaciones favorables y se eliminan las desfavorables...".

“...Esta competencia es más intensa entre los organismos de la misma especie pues tienen las mismas necesidades. Sobreviven quienes ganan la competencia..."

Asumida como una visión en la cual se asigna un propósito a los organismos y a fenómenos naturales.

Entendido como el acto de poner en contacto los sentidos con una realidad. 
En L3 se presenta la siguiente afirmación:

“...Los organismos que sobreviven son los que presentan la combinación de caracteres idóneos para hacer frente al ambiente (clima, competidores, enemigos, etc.)...".

\section{Categoría Observación}

En el L1 se afirma “... Al observar los criaderos de animales, Darwin se percató de que mediante varias generaciones de cruces controlados, un criador podía obtener un rebaño de animales con ciertas características deseadas..." Frase que puede llevar a los estudiantes a pensar que se hace ciencia observando, lo cual puede hacer que se creen imaginarios erróneos de lo que significa la ciencia y de cómo se construye el conocimiento científico. Camilloni (1997) afirma que la búsqueda prematura de este tipo de afirmaciones, a partir de las denominadas "experiencias cruciales", inducen al alumno a pensar que la ciencia se construye de manera concluyente, sobre la base de experiencias simples, lineales y acumulativas.

En el L2 se plantea “... Charles Darwin (1809-1882) naturalista ingles, inició su teoría sobre el origen de las especies, a partir de sus observaciones realizadas en América del Sur, en las islas Galápagos y otras regiones...", en este planteamiento también está la idea que el discurso de la evolución surge a través de la observación y no se pone de presente que existieron toda una serie de preguntas e inquietudes acerca del origen de los seres vivos y de explicaciones que en la época se daban en cuanto a los fenómenos que ocurrían en éstos. Si bien la observación jugó un papel importante no fue el único elemento que llevó a Darwin a plantear la teoría de la evolución, también estuvo respaldada en estudios taxonómicos e interpretación de los planteamientos de otros autores. En este sentido Bachelard, hace una crítica al empirismo clásico y a su afán por construir generalizaciones a partir de lo que observa.

Estos planteamientos pueden llevar al estudiante a generar un obstáculo de experiencia básica, en el cual se induce a pensar que a través del sentido de la observación se conoce los fenómenos tal y como son; imposibilitando acceder al conocimiento científico, debido a que éste se vuelve científico sólo cuando se le somete a la crítica de la primera impresión, por lo tanto es necesario que el estudiante tenga conocimiento de lo limitado que resultan los sentidos al momento de conocer.

Estas frases dejan ver un rezago de intencionalidad en la interpretación que el autor hace de la teoría de la evolución, esto podría ser producto de una cosmovisión finalista la cual circula todavía en los discursos educativos, puesto que ha hecho parte de la cultura y por consiguiente de los conocimientos previos que se puedan tener frente a una teoría tan paradigmática como es ésta; hecho que sin duda alguna permea los libros de texto ya que éstos son realizados por autores que como sujetos sociales están cargados de todo un legado cultural el cual consciente o inconsciente influye en la interpretación que tiene del tema.

Lo anterior puede llevar a quienes hacen uso de los textos a dos tipos de obstáculos por un lado el animista dado que se le otorgan características finalistas o dotadas de propósito a ciertos hechos de la naturaleza y por otra parte al obstáculo de experiencia básica, ya que se conoce un hecho a partir de la interpretación que otro ha hecho previamente, en este caso el autor del texto. 


\section{Recomendaciones acerca del uso de textos escolares en referencia al tema de evolución por selección natural}

Al momento de elegir y usar los libros de texto es importante que el maestro sea crítico y a la vez incentive posturas críticas en los estudiantes, en relación a los contenidos y afirmaciones que se presentan en estos.

Es importante que los docentes identifiquen el poco manejo que en los libros de texto se hace de las fuentes bibliográficas ya que una de sus tareas debe ser ayudar a comprender a los estudiantes que lo presentado en los libros es producto de una transformación que realizan las editoriales de los escritos originales y de otro tipo de textos.

El conocimiento se presenta a través de forma que no fomenta una actitud critica en los estudiantes, ya que es en su mayoría es presentado como una afirmación que no dan lugar a refutaciones. En este sentido, el maestro tiene un papel importante debido a que éste debe hacer las aclaraciones pertinentes para hacer ver a los estudiantes que el conocimiento es dinámico.

El papel del maestro al momento de usar los libros de textos es fundamental, ya que es a él quien le corresponde hacer las aclaraciones necesarias a partir de las incomprensiones o dificultades que surjan en los estudiantes al momento de hacer uso de este recurso educativo.

Es recomendable que a nivel educativo se trabaje la evolución como un pensamiento transversal más que como una temática particular.

\section{Conclusiones:}

- Los planteamientos erróneos o imprecisos encontrados con mayor frecuencia en los libros revisados son los de conocimiento general y el animista.

- Las afirmaciones y las generalizaciones pueden llevar a los estudiantes a la generación de obstáculos epistemológicos, induciendo a pensar que la ciencia se construye: de manera concluyente, sobre la base de experiencias simples o de manera lineal y acumulativa; lo cual contribuye a inmovilizar el pensamiento de los estudiantes.

- Un análisis de la historia del pensamiento evolutivo, puede ayudar a identificar algunos conceptos o representaciones que constituyeron obstáculos epistemológicos al momento de entender el tema de la evolución por selección natural. Este análisis puede sugerir la existencia de obstáculos similares en el proceso de construcción individual, sin que ello implique una recapitulación individual del proceso histórico de construcción del conocimiento.

- Es importante que el maestro conozca cuales son los obstáculos epistemológicos más frecuentes en los estudiantes a la hora de abordar la teoría de la evolución por selección natural, para que así pueda orientar los procesos de enseñanza de esta teoría. 
- El tema de evolución por selección natural tiende a ser simplificado en los libros de texto usados en este estudio. En este sentido se podría decir que cuanto mayor es la complejidad de la teoría y mayor fragmentación hay en el discurso utilizado, más se corre el riesgo de generar un obstáculo de tipo epistemológico.

- Se encontró que los autores de los libros al plasmar sus ideas, en ocasiones erróneas, pueden propiciar dificultades en los estudiantes al momento de abordar la temática de la evolución por selección natural. Por tanto, es importante que los autores tengan presente la complejidad de la teoría evolutiva al momento de adaptarla a los libros de texto.

- Se encontró, en los libros de texto analizados, una tendencia a reducir a un tema especifico el pensamiento evolutivo.

- Los libros trabajados (L1), (L2) y (L3) presentan diferencias en la frecuencia de aparición de planteamientos inadecuados. Se encontró que los libros (L1) y (L2) presentan más planteamientos inadecuados al momento de abordar la teoría de la evolución por selección natural que el libro (L3).

- La técnica del análisis de contenido utilizada en este trabajo fue pertinente, debido a que facilitó la identificación de los planteamientos inadecuados, presentes en libros de texto al momento de exponer la teoría de la evolución por selección natural.

\section{BIBLIOGRAFÍA}

- Arnal, J. Rincón, D. (1992). Investigación educativa: fundamentos y metodologías. España. ed. Labor.

- Audesirk, G, Audesirk T, Byers, B. (2003) Biología. La vida en la Tierra. México, Editorial Pearson Educación.

- Alzate, M. (2000). Cómo leer un texto escolar: texto, paratexto e imágenes. Revista ciencias humanas. Colombia, $\mathrm{N}^{\circ} 20$, mayo.

- Bachelard, G. (1948). La formación del espíritu científico. ed 12․ Buenos Aires: siglo XX1.

- Bidau, C. (2001) La enseñanza de la biología evolutiva en la escuela. Memorias de las V Jornadas Nacionales de Enseñanza de la Biología. La educación en biología: tendencias e innovaciones. Argentina.

- Badii, M., Landeros, J., Garza, V. (2008) Historia evolutiva de la tierra. CULCyT//Enero Febrero, 2008 Año 5, $\mathrm{N}^{\circ} 24$.

- Cambell, N., Mitehell, L., Reece, J. (2001) Biología conceptos y relacionas. Editorial Pearson Educación, México. Tercera Edición.

- Camilloni, A. (1997). Los obstáculos epistemológicos en la enseñanza. Barcelona ed Gedisa. 
- Cuellar, L., Pérez, R., Quintanilla, M. (2005) la propuesta de Ernest Rutherford en los libros de texto en Colombia, un análisis desde la historia de las ciencias y la visión de transposición didáctica en ellos. Enseñanza de las ciencias. 2005, número extra. Vll congreso.

- Curtis, H. Barnes, S. Schenek, A. (2008). Masarini A. Biología. 7 Edición, Buenos Aires, editorial médica panamericana.

- Dobzhansky. T., Ayala, F., Stebbins G. y Valentine, J. (1980). Evolución. Barcelona: Omega.

- Jacob, F. (1986).La lógica de la viviente. Biblioteca científica. Salvat.

- García, C. (1998).Los conceptos estructurantes: una aproximación a la enseñanzaaprendizaje de la geología. Enseñanza de las ciencias vol (16) 2 pp. 223-230.

- Harris, L. (1985).Evolución, génesis y revelaciones. Ciencias de la naturaleza C.

- Hernández, G. (2003). Abatir los obstáculos en el aprendizaje de matemáticas y ciencias. Memorias de la novena reunión de análisis de la actividad experimental en el aprendizaje de las ciencias naturales y exactas. Culiacán,. Esteros Editores.

- Jiménez, A., Torres, A. (2004). La práctica investigativa en ciencias sociales. Universidad Pedagógica Nacional.

- Mayr, E (1980) The evolucionary sinthesis: perspectives on the unification of biology. Harvard University Press. Massachusetts.

- Mayr, E (2006) ¿Por qué es única la biología? Consideraciones sobre la autonomía de una disciplina científica ed. Buenos Aires.

- Mayr, E (2005). Así es la biología. España, Editorial Debate.

- Monje, J., Gómez, P., Rivas, M. (2005) Biología general. Editorial universidad estatal a distancia. San José de Costa Rica.

- Mora, A (2005).Obstáculos epistemológicos que afectan el proceso de construcción de conocimientos en el área de ciencias en niños de edad escolar. Disponible en:

\section{http://www.cientec.or.cr/exploraciones/ponenciaspdf/arabelamora2.pd.}

- Piñuel, J. (2002). Epistemología, metodología y técnicas del análisis de contenido. Universidad Complutense de Madrid. Estudios Sociolingüístico.

- Rojas, O. Tiempo geológico. (2008) Universidad de concepción. Facultad de arquitectura, urbanismo, geografía. Departamento de geografía. Ciudad universitaria, concepción. Chile. Disponible en: http://www.udec.cl/ ocrojas

- Bunge, M. Diccionario de filosofía. (2005) Siglo XXI editores S.A. México D. F.

- Ayala, F. (2006). La evolución de un evolucionista. Colleció Honoris Causa. Universidad de Valencia. España. 


\section{BIBLIOGRAFIA DE APOYO}

- Alvarado, J. Varela J. (2006) La importancia del uso del libro de texto en el proceso de enseñanza aprendizaje en el bachillerato universitario. Akademeia, vol 4, n. 6. octubrediciembre.

- Ayuso, E. y Banet, E. (2002). Pienso más como Lamarck que como Darwin: comprender la herencia biológica para entender evolución. Alambique 32.

- Bar, A. y Valenzuela, S. (2004). "Estrategias metodológicas y errores conceptuales que afectan la comprensión de la noción de evolución en textos de biología para el nivel polimodal". Universidad Nacional del Noredeste, comunicaciones científicas y tecnológicas.

- Bar, A. y Valenzuela, S. (2006). "La evolución como modelo de explicación en los libros de textos escolares", Cinta de Moebio, septiembre, número 026, universidad de Chile, Santiago de Chile.

- Castro, A. (2003).Los libros de texto: filosofía e historia de las ciencias. Nodos y nudos, vol. 2, no 15 , julio-diciembre.

- Cummins, C. y Demastes, S. (1994). Evolution: biological education`s under-researched unifying theme. Journal of research in science teaching 31 (5), pp. 445-448.

- Futuyma, D (1986). Evolutionaty biology. Second Edition. Traducido y adaptado por: Rodrigo Torres, Universidad Pedagógica Nacional.

- Mateu, M. (2005) Libros escolares para ciencias naturales en el tercer ciclo de la EGB. Enseñanza de las ciencias, número extra, Vll congreso. Argentina. Universidad nacional de general San Martín. Mayr, E. (2006) ¿Por qué es única la biología? Consideraciones sobre la autonomía de una disciplina científica ed. Buenos Aires.

- Mayr, E (1992) Una larga controversia: Darwin y el Darwinismo. Drakontos, España.

- _ (1988) Towards a new philosophy of biology.Observations of an evolutionist. Cambridge: Harvard University Press

- Solari, A. (2004).Genética humana fundamentos y aplicación en medicina, editorial panamericana, 3 edición.

- Soler, M. (2003). Evolución. La base de la biología. Granada: proyecto sur de ediciones.

- González, G. y Meinardi, E. (2005).Tercer encuentro de investigadores en didáctica de la biología, estudio de las concepciones acerca de la teoría de la evolución en estudiantes, profesores y licenciados en biología. 